\title{
Metathesis of 9, 12-Octadecenoic Acid Methyl Ester: A Potential Source for Industrially Important Platform Chemicals
}

\author{
Yelchuri Vyshnavi ${ }^{1}$, Rachapudi Badari Narayana Prasad ${ }^{2}$, Mallampalli Sri Lakshmi Karuna ${ }^{3}$ \\ Centre for Lipid Research, CSIR- Indian Institute of Chemical Technology, Hyderabad 500 007, India
}

\begin{abstract}
With the increase in environmental concern the demand for renewable sources and modern, greener synthetic routes is growing day by day. A number of self and cross-metathesis reactions were carried out across the 9-octadecenoic acid, and different vegetable oils, to develop a range of organic intermediates useful for lubricants, plasticizers, cosmetics, and grease applications. However, there is no systematic data available on the metathesis of fatty acids with more than one double bond. In this context, the present study was focused on the use of olefin metathesis for the preparation of industrial platform chemicals from 9,12-octadecenoic acid methyl ester employing Grubb's second generation catalyst, $0.03 \mathrm{~m}$ mol concentration at $40-45^{\circ} \mathrm{C}$ for $36 \mathrm{~h}$. 6-pentadecene (5\%), and cyclic hydrocarbon cyclodecacyclododecene (19\%) were observed in major amounts. Mono esters namely cyclopropaneoctanoic acid methyl ester (12\%) and pentadecenoic acid methyl ester (11\%) were also observed, which may have potential as synthetic organic intermediates.
\end{abstract}

Keywords: Self-metathesis, Grubb's second generation catalyst, 9,12-octadecenoic acid methyl ester , Synthetic organic intermediates, Cyclodecacyclododecene

\section{Introduction}

Olefin-metathesis has emerged as a powerful tool over the past decades for the formation of $\mathrm{C}-\mathrm{C}$ bonds [1]. The reactions have been extensively used in organic and polymer chemistry for the development of number of platform chemicals [2]. Linear $\alpha$, $\dot{\omega}$-dicarboxylic acids such as suberic acid [3] which are derived by the metathesis of unsaturated fatty acids form the important building blocks in the synthesis of materials like nylons, cosmetics, plasticizers, lubricants and greases. The long chain linear $\alpha$, $\omega$ dicarboxylic acids with better performance properties can be generated employing metatheis route, which are otherwise difficult to be produced by conventional methods.

Vegetable oils and fats with long chain unsaturated fatty acids are potential sources, where the chemical modifications across the double bond results in a variety of organic intermediates [4]. Apart from dicarboxylic acids, metathesis of long chain unsaturated fatty acids with more than one double bond results in a wide variety of intermediates $[5,6]$ and value added products. The development of oleochemistry towards fine chemicals has been carried out employing a variety of homogeneous and heterogeneous catalysts today [7]. Although the catalytic systems like homogeneous catalysts $\mathrm{WCl}_{6}-\mathrm{Me}_{4} \mathrm{Sn}$ and heterogeneous catalysts $\mathrm{Re}_{2} \mathrm{O}_{3}-\mathrm{Al}_{2} \mathrm{O}_{3}[8,9]$ gave high selectivity and high conversions, they suffered from low catalyst turnover numbers. Further the catalysts were also found to be moisture sensitive, on the other hand, Grubbs' catalysts first and second generation were found to be most stable, sensitive to oxygen and particularly in aqueous medium [10]. A number of reports have been published on self and cross metathesis of vegetable oils $[11,12]$ and fatty acids [13] employing Grubbs' catalysts.

However, in case of metathesis of fatty acids, there is no systematic data available on the metathesis of unsaturated fatty acids with more than one double bond. Though, there exists a report on the metathesis of 9, 12-octadecenoic acid methyl ester and metathesis of vegetable oils with good amounts of cyclodecacyclododecene, there is no report available on the generation of different products and the mechanism of their formation [14]. Hence in the, the present study of the self-metathesis of pure 9,12-octadecenoic acid methyl ester (99\%) with two double bonds employing Grubbs' second generation catalyst was carried out to understand the mechanism of product formation. The various metathesized products formed during the reaction were identified using GC and GC-MS. Also, the study gives a detailed mechanism involved for the generation of various metathesized products.

\section{Materials and Methods}

\subsection{Materials}

Linoleic acid methyl ester (99\%), sulfuric acid, dry methanol, dry dichloromethane (99.9\%) and tricyclohexylphosphine [1,3-bis(2,4,6-trimethylphenyl)-4,5dihydroimidazol-2-ylidene benzylidene ruthenium (IV) dichloride (Grubb's second generation catalyst) (II), were purchased from M/s Sigma Aldrich Chemical Co, Hyderabad and were used as received.

\subsection{Methods}

\subsubsection{Self-metathesis of linoleic acid methyl ester}

linoleic acid methyl ester $(2.00 \mathrm{~g}, 6.7 \mathrm{mM})$ was dissolved in $20 \mathrm{ml}$ dry DCM taken into a two necked round bottom flask under $\mathrm{N}_{2}$ atmosphere. Grubb's second generation catalyst $(0.026 \mathrm{~g}, 0.03 \mathrm{mmol})$ taken in dry DCM was added to the methyl esters using a $20 \mathrm{ml}$ syringe. The contents were heated at $40-45^{\circ} \mathrm{C}$ for $15 \mathrm{~h}$. The reaction was monitored by TLC eluted with hexane:ethyl acetate $(90: 10, \mathrm{v} / \mathrm{v})$. The contents were washed with ethyl acetate and passed over 


\section{International Journal of Science and Research (IJSR) \\ ISSN (Online): 2319-7064}

Index Copernicus Value (2013): 6.14 | Impact Factor (2014): 5.611

anhydrous sodium sulphate. The solvent was removed using rotary evaporator and dried under reduced pressure $(2-5 \mathrm{~mm}$ $\mathrm{Hg})$ to obtain metathesized products $(0.140 \mathrm{~g}, 70 \%$ yield $)$. The products were qualitatively and quantitatively identified using GC and GC-MS.

\subsubsection{Gas Chromatography analysis}

The linoleic acid methyl esters and the products obtained after self-metathesis were analyzed using an Agilent 6890 Gas Chromatograph (Agilent Technologies, Palo Alto, CA, USA) fitted with an FID detector and split/splitless injector. A non-bonded cyano silicone column (DB-225, $30 \mathrm{~m} \times 0.32$ i.d., J\&W Scientific, USA) was employed for Gas Chromatography analysis. The column temperature was initially maintained at $60{ }^{\circ} \mathrm{C}$ for $2 \mathrm{~min}$, increased to $300{ }^{\circ} \mathrm{C}$ for a hold time of $20 \mathrm{~min}$ with a flow rate of $10{ }^{\circ} \mathrm{C} / \mathrm{min}$. The injector and detector temperatures were set at $250{ }^{\circ} \mathrm{C}$ using Chemstation software for the data analysis (Fig. 1).



\subsubsection{GC-MS Analysis}

Self-metathesis of linoleic acid methyl ester was analyzed using an Agilent $6890 \mathrm{~N}$ (Agilent Technologies, Palo Alto, CA, USA) Series equipped with an DB-225 Column (30 m x $0.25 \mathrm{~mm}$ i.d) series Gas Chromatograph connected to an Agilent 5973. Mass Spectrometer operating in the EI mode $\left(70 \mathrm{eV} ; \mathrm{m} / \mathrm{z}\right.$ 50-550; source temperature $230{ }^{\circ} \mathrm{C}$ and a quadruple temperature $150{ }^{\circ} \mathrm{C}$ ). The column temperature was initially maintained at $100{ }^{\circ} \mathrm{C}$ for $2 \mathrm{~min}$, increased to $300{ }^{\circ} \mathrm{C}$ at $10{ }^{\circ} \mathrm{C} / \mathrm{min}$ with a hold time of $20 \mathrm{~min}$ at $300{ }^{\circ} \mathrm{C}$. The inlet temperature was maintained at $300{ }^{\circ} \mathrm{C}$ and split ratio of 50:1. Structural assignments were based on interpretation of mass spectrometric fragmentation and confirmed by comparison of retention times as well as fragmentation pattern of authentic compounds and the spectral data obtained from the Wiley and NIST libraries (Fig. 2).

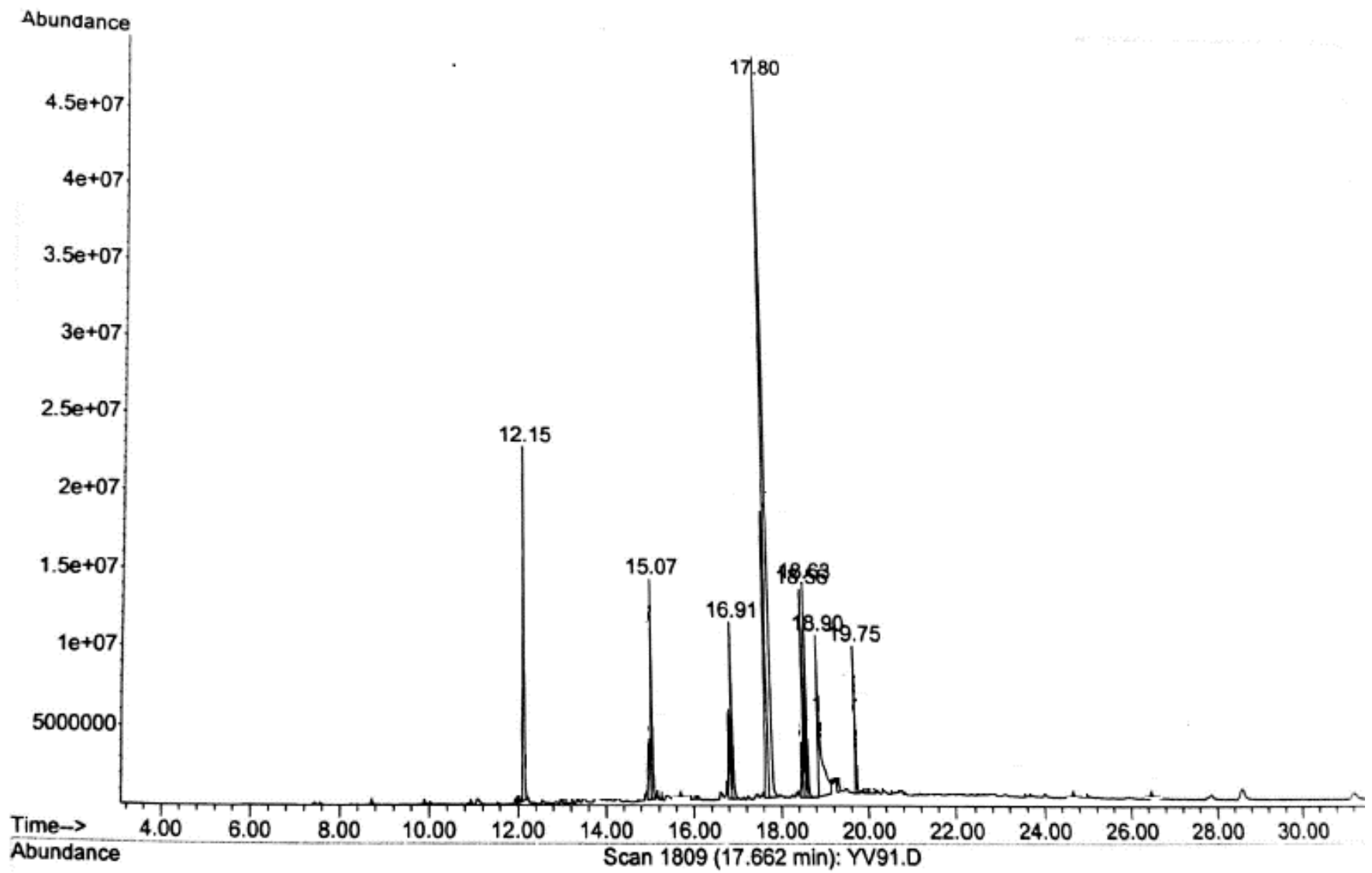

Fig. 2. GC-MS analysis of self-metathesis of linoleic acid methyl ester at $0.03 \mathrm{mmol}$ concentration 


\section{International Journal of Science and Research (IJSR) \\ ISSN (Online): 2319-7064}

Index Copernicus Value (2013): 6.14 | Impact Factor (2014): 5.611

\section{Results and Discussions}

Self-metathesis of 9,12-octadecenoic acid methyl ester (99\%) employing Grubbs' second generation catalyst resulted in unsaturated acyclic and cyclic hydrocarbons and unsaturated monoesters. 6-pentadecene (Ret. time-12.7 min, $5 \%$ GC; m/z 210, GC-MS, Fig. 3), a type of alarm pheromone found in acarid mite was observed in minor amounts. Cyclic hydrocarbon such as cyclodecacyclododecene (Ret. time-21.34 min, 19\% GC; m/z 260, GC-MS, Fig. 4), cyclopropane octanoic acid methyl ester (Ret.time-13.09 $\mathrm{min}, 12 \%$ GC; m/z 200, GC-MS, Fig. $5)$, were also observed in minor amounts. Similar products were observed in our earlier study on self-metathesis of rubber fatty acid methyl esters [6]. These may have potential as synthetic intermediates in organic synthesis, Fig. 3 shows the mechanism of formation of these products. Another unsaturated ester, 9-pentadecenoic acid methyl ester (Ret.time: $16.9 \mathrm{~min}, 11.12 \% \mathrm{GC} ; \mathrm{m} / \mathrm{z}$ 254, GC-MS, Fig. 3), was also observed during the reaction which is an unusal chain length containing molecule and is difficult to be prepared employing conventional method. This was also observed in our earlier study on self-metathesis of palash fatty acid methyl esters [14]. Fig. 3 Shows the mechanism involved in the formation of the above metathesized products. Thus, the study reports for the first time a systematic data on the self-metathesis of 9,12-octadecenoic acid methyl ester.

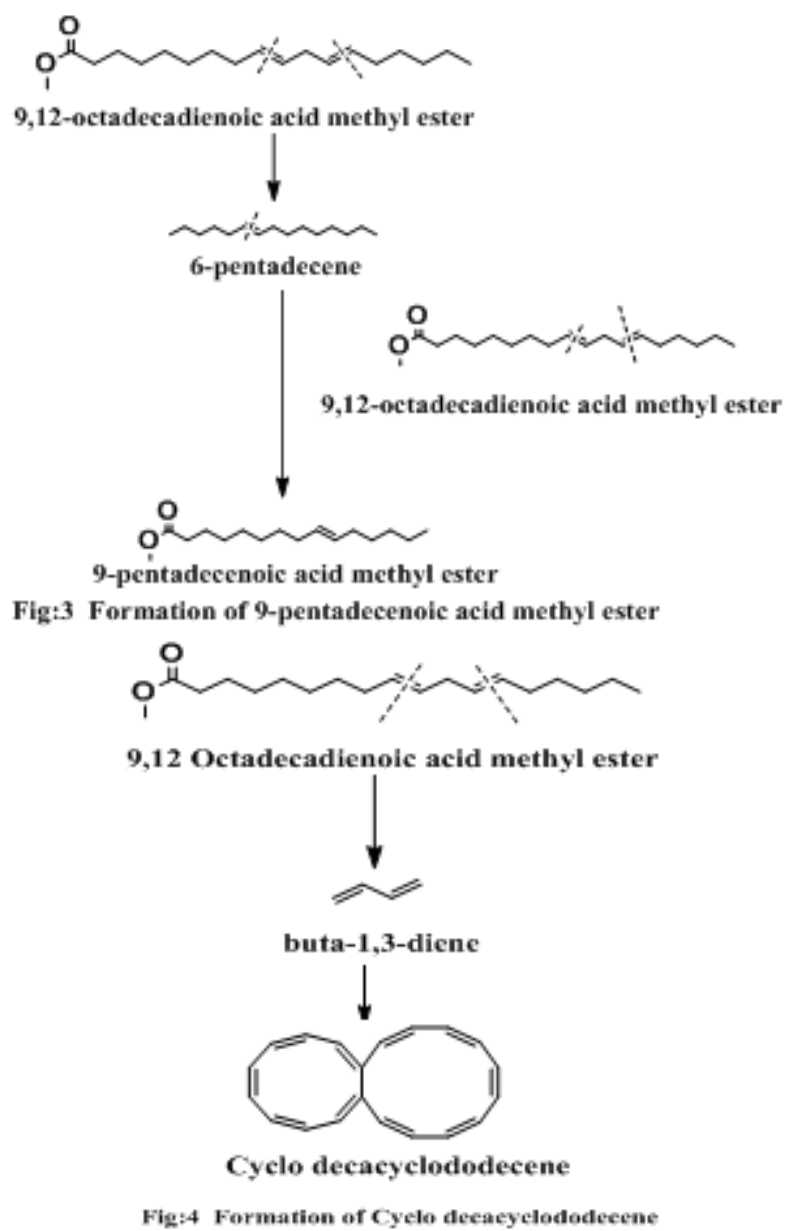

\section{Conclusions}

A detailed study on metathesis of 9,12-octadecenoic acid methyl ester was carried out employing grubbs' second generation catalyst. The reaction resulted in mono ester, cyclopropaneoctanoic acid methyl ester and pentadecenoic acid methyl ester; cyclic hydrocarbons, cyclodecacyclododecene which have potential as synthetic intermediates. The study revealed for the first time, a detailed mechanism involved in the formation of various metathesized products for 9,12-octadecenoic acid methyl ester. This helps the chemist to synthesize any of the above mentioned products by the metathesis of 9,12-octadecenoic acid methyl ester employing metathesis route, which are otherwise difficult to be synthesized employing conventional route.

\section{Acknowledgements}

This work was carried with the financial grant given by CSIR and was supported by AcSIR-IICT, India.

\section{References}

[1] K.J. Ivin, J.C. Mol, "Olefin Metathesis and Metathesis Polymerization," Academic Press: San Diego, CA. 1997.

[2] P. Schwab, R. H Grubbs, J. W. Ziller, “A Series of Well Defined Metathesis Catalysts-Synthesis of [RuCl2(CHR)(PR3)2] and Its Reactions," J. Am. Chem. Soc. 118, 100, 1996.

[3] L. Ngo Helen, Kerby Jones, and Thomas A. Foglia. "Metathesis of Unsaturated Fatty Acids: Synthesis of Long-Chain Unsaturated- $\alpha, \omega$-Dicarboxylic Acids," JAOCS, 83, (7), 2006.

[4] B.B. Marvey, "Sunflower-based feedstocks in non-food applications: perspectives from olefin metathesis,'Int. J. Mol. Sci. 9, 1393, 2008.

[5] Yelchuri Vyshnavi, R. B. N. Prasad, M. S. L. Karuna, "Metathesis of tobacco fatty acid methyl esters: Generation of industrially important platform chemicals," Ind. Crops Prod. 50,701, 2013.

[6] Yelchuri Vyshnavi, R. B. N. Prasad, M. S. L. Karuna, Metathesis of Rubber Fatty Acid Methyl Esters: A 
Green Approach for Generating Industrial Platform Chemicals IJSR. 5 (3), 209, 2014.

[7] B.B. Marvey, J. A. K. Du-Plessis, H. C. M. Vosloo, J. C. Mol, "Metathesis of unsaturated fatty acid esters derived from South African sunflower oil in the presence of a 3 wt\% Re2O7/SiO2-A12O3/SnBu4 catalyst,"J. Mol. Catal. A: Chem. 201, 297, 2003.

[8] U. Biermann, W. Friedt, S. Lang, W. Luhs, G. Machmuller, J.O. Metzger, G.K.M. Rusch, H.J. Schafer, M.P. Schneider, "New Syntheses with Oils and Fats as Renewable Raw Materials for the Chemical Industry". Angew. Chem. Int. Ed. Engl. 39, 2206, 2000.

[9] S.Z. Erhan, M.O. Bagby, T.C. Nelsen, "Drying properties of metathesized soybean Oil," J. Am. Oil Chem. Soc. 74, 703-706, 1997.

[10] L.D.M.S. Kanaoka, R.H. Grubbs, 1996. Living ringopening metathesis polymerization in aqueous media catalyzed by well-defined ruthenium carbene complexes. J. Am. Chem. Soc. 118, 784, 1996.

[11] V.B. Veljkovic, S.H. Lakicevic, O.S. Stamenkovic, Z.B. Todorovic, M.L. Lazic, "Biodiesel production from tobacco seed oil with high content of fatty acids". Fuel. 85, 2671, 2006.

[12]M.D. Refvik, R.C. Larock, Tian, Q. "Rutheniumcatalyzed metathesis of vegetable Oils," J. Am. Oil Chem. Soc. 75, 93-98, 1999.

[13] J.C. Mol, "Catalytic metathesis of unsaturated fatty acid esters and oils". Top Catal. 27, 97-104, 2004.

[14] Yelchuri Vyshnavi, Rachapudi Badari Narayana Prasad and Mallampalli Sri Lakshmi Karuna, "Synthesisof industrially important platform chemicals viaolefin metathesis of palash fatty acid methyl esters". European Journal of Chemistry 5 (3) 532-535, 2014 . 


\section{International Journal of Science and Research (IJSR) \\ ISSN (Online): 2319-7064}

Index Copernicus Value (2013): 6.14 | Impact Factor (2014): 5.611

Author Profile

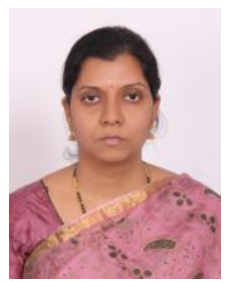

Dr M.S.L. Karuna obtained her (M. Sc.) in organic chemistry and $\mathrm{Ph} \mathrm{D}$ (Lipid Chemistry) from Osmania University. She has 17 years of research experience and presently holding the Senior Scientist position at Centre for Lipid Research. Her research expertise is in the areas of chemo-enzymatic synthesis of oleochemicals and bioactives and nutraceuticals. She has 45 publications in both national and international journals and has 9 patents ( 4 granted and 5 filed). She has received Associate Fellow of Andhra Pradesh Akademi of Sciences, 2015; S.D. Tirumala Rao Award, 2014; IICT Best Woman Scientist Award, 2011conferred by IICT; R.K. Khanna Memorial Award, 2009; OP Narula Technology Award, 2005 \& 2014 and OP Narula Young Scientist Award, 2004 by Oil Technologists' Association of India (OTAI), Southern Zone. 